\title{
Follow-Up CT Results of COVID-19 Patients with Initial Negative Chest CT
}

This article was published in the following Dove Press journal:

Infection and Drug Resistance

\author{
Binjie Fu $\mathbb{D}^{1, *}$ \\ Liangbo $\mathrm{Hu}^{2, *}$ \\ Fajin Lv' \\ Junhao Huang ${ }^{2}$ \\ Wangjia Li (iD) \\ Yu Ouyang' \\ Zhigang Chu (D)
}

'Department of Radiology, The First Affiliated Hospital of Chongqing Medical University, Chongqing, People's Republic of China; ${ }^{2}$ Department of Radiology, Yongchuan Hospital of Chongqing Medical University, Chongqing, People's Republic of China

*These authors contributed equally to this work
Correspondence: Zhigang Chu Department of Radiology, The First Affiliated Hospital of Chongqing Medical University, I\# Youyi Road, Yuanjiagang,

Yuzhong District, Chongqing 400016,

People's Republic of China

Tel +8618723032809

Fax +86 2368811487

Email chuzg0815@I63.com
Purpose: To determine whether new pulmonary lesions will develop in COVID-19 patients with negative initial chest CT findings and to investigate their CT features and outcome during treatment.

Patients and Methods: Data were collected retrospectively from 29 patients who had tested positive for COVID-19 by reverse-transcription polymerase chain reaction testing but negative by initial chest CT from January 22 to February 17, 2020. Clinical manifestations, laboratory indicators, and follow-up CT data were evaluated.

Results: Among 317 confirmed COVID-19 patients, 29 (9.1\%) (mean \pm SD, $38.5 \pm 20.5$ years; 12 women) with negative initial chest CT findings were evaluated. New pulmonary lesions developed in $10(34.5 \%)$ patients on follow-up CT. Mean time from onset of new lesions to initial CT was $5.8 \pm 3.0$ days (range: $2-12$ days). New lesions (mean involved lobes and segments: $2.5 \pm$ 1.6 [range: 1-5] and $4.5 \pm 4.5$ [range: 1-13]) were mainly spherical/patchy ground-glass opacities frequently located in the left lower lobe $(9,90.0 \%)$. Among the 10 patients, lesions in $6(60.0 \%)$ indicated progression after occurrence, and those in $10(100.0 \%)$ indicated significant absorption on latest CT. When new lesions developed, $6(60.0 \%)$ patients developed new symptoms or had aggravated symptoms and 3 (30.0\%) had decreased lymphocyte count. Patients with worsening symptoms had higher involvement of lung segments (mean: $6.5 \pm 5.0$, range: $1-13)$ than asymptomatic patients (mean: $1.5 \pm 0.6$, range: $1-2)(\mathrm{P}=0.057)$.

Conclusion: In COVID-19 patients with negative initial chest CT findings, new pulmonary lesions may develop during treatment. Repeat CT is necessary for monitoring the disease, especially when patients have worsening symptoms or laboratory indicators.

Keywords: coronavirus disease 2019, SARS-CoV-2, follow-up, CT imaging

\section{Introduction}

Coronavirus disease 2019 (COVID-19) is a global, high-risk acute respiratory infectious disease caused by severe acute respiratory syndrome coronavirus 2 (SARS-CoV -2) infection. ${ }^{1-3}$ Because the receptor that mediates SARS-CoV-2 invasion into human host cells is the same as the angiotensin-converting enzyme 2 receptor for human SARS-CoV, it naturally determines its method of transmission. ${ }^{4,5}$ The entire population is generally susceptible, and elderly individuals and those with underlying diseases typically experience more severe symptoms. ${ }^{6}$ Because of its strong transmission ability and long incubation period, ${ }^{7,8}$ the disease has spread rapidly internationally. As of March 30, 2020, the pandemic has spread to 202 countries and regions, and a total of $1,521,252$ cases have been diagnosed, resulting in 92,798 deaths. ${ }^{9}$

COVID-19 is confirmed by reverse-transcription polymerase chain reaction (RTPCR) or genetic sequencing of respiratory/blood samples. ${ }^{10,11}$ However, cases with 
suspected false-negative results remain a serious problem because of limited sensitivity, shortage of supplies, and relatively long processing time of the new coronavirus nucleic acid kit. Consequently, the timeliness of patients receiving treatment is affected. CT is one of the most common methods for pneumonia diagnosis, and it can quickly and efficiently provide additional information about pulmonary lesions. The advantages of CT detection can compensate for the disadvantages of RT-PCR, but the results of $\mathrm{CT}$ detection may be inaccurate in the early stage of the disease. ${ }^{12}$ Therefore, continuous follow-up CT should be conducted to accurately observe and follow up the patient's condition.

Notably, a study indicated that chest CT manifestations of COVID-19 may appear prior to negative RTPCR results. ${ }^{13}$ However, in clinical practice, we also found that some patients were positive for viral nucleic acid, but no abnormality was found on initial chest CT. Such cases are common in screening high-risk populations. For these patients, no study has examined whether new lesions will develop during treatment and their outcomes or whether new developed lesions can be reflected by the patients' clinical manifestations or laboratory results.

This study aimed at reviewing the follow-up CT of COVID-19 patients with negative initial chest CT findings while evaluating changes in clinical manifestations and laboratory indicators of patients with newly developed lesions and studying the correlation of clinical and laboratory data with imaging results. The results will allow radiologists and clinicians to better understand the conditions of pulmonary lesions in these special patients.

\section{Patients and Methods}

This study was approved by the Ethics Committee of the First Affiliated Hospital of Chongqing Medical University (No. 2020-210).

\section{Patients}

From January 22, 2020, to February 17, 2020, a total of 317 patients with confirmed COVID-19 were admitted to 2 hospitals in 2 districts in Chongqing city, China. Of these patients, 29 (9.1\%) (17 men, 12 women; $38.5 \pm$ 20.5 years, range $4-73$ years) who had initial negative chest $\mathrm{CT}$ were included in this study. All patients tested positive for respiratory secretions obtained by bronchoalveolar lavage, tracheal aspirate, nasopharyngeal swab, or oropharyngeal swab by real-time RT-PCR. Patient selection in both institutions for this study was consecutive, and no exclusion criteria were applied. Besides sex and age, clinical information was also collected. In addition, close follow-up (follow-up time window was determined based on clinical evaluation) of patients was conducted after treatment, and changes in patients' clinical manifestations, laboratory indicators, and chest $\mathrm{CT}$ scans were recorded.

\section{Chest CT Examination}

All patients underwent a noncontrast $\mathrm{CT}$ when admitted to the hospital. Repeat follow-up CT scans for each patient were performed. The chest CT scans were performed using SOMATOM Emotion 16 (Siemens Healthineers, Erlangen, Germany), Aquilion ONE (Toshiba Medical Systems, Tokyo, Japan), Philips Ingenuity Core 128 (Philips Medical Systems, Best, Netherlands), and SOMATOM Definition AS (Siemens Healthineers, Erlangen, Germany) spiral CT scanner. All CT scans were acquired with the patients in the supine position at the end of inspiration without contrast and with both upper limbs raised above the head. The scanning range was from the thoracic entrance to the costophrenic angle. Other acquisition parameters were as follows: tube voltages: 110-130 $\mathrm{kV}$, with automatic tube current; scanning slice thickness and slice spacing: $5 \mathrm{~mm}$; and matrix: $512 \times 512$. After scanning, the images were reconstructed to $1 \mathrm{~mm}$ and transferred to a picture archiving and communication system.

\section{CT Image Analysis}

Two senior radiologists ( $\mathrm{Lv}$ and $\mathrm{Chu}$ ) specializing in chest imaging, who were blinded to the patients' information, independently evaluated the initial and followup CT images of all patients. The first step was to determine which patient developed new pulmonary lesions. Subsequently, the distribution, distribution type, involved scope, and imaging characteristics of the newly developed lesions were observed and recorded. The evaluation indicators were as follows: 1) distribution of lesions (unilateral lung or bilateral lungs, involved lobes and segments), 2) involved scope (the number of involved lobes and segments), 3) the shape of the lesions (round or patchy), 4) density (ground-glass opacity [GGO] or consolidation), 5) uniformity of density (homogeneous or heterogeneous), and 6) the outcome of lesions on repeat CT scans 
(decrease or increase in size and density). The results were based on the agreement of the opinions of the two physicians.

\section{Statistical Analysis}

All data were statistically analyzed using SPSS 20.0 (SPSS III, Chicago, USA). Data were expressed as mean \pm standard deviation for continuous variables and as numbers and percentages for categorical variables. In the comparison of clinical and laboratory characteristics and their changes between patients with and without new pulmonary lesions, the Fisher test and Mann-Whitney $U$ test were used for categorical variables, and the Student's $t$-test was used for continuous variables (age and number of lung segments). A p-value $<0.05$ was considered statistically significant.

\section{Results}

\section{Patients' Basic Conditions}

The basic clinical and laboratory characteristics of the 29 patients with COVID-19 are listed in Table 1. Overall, there were more male patients than female patients. Most patients had a history of contact with confirmed COVID-19 patients but did not have a long residence in the main epidemic area. The main clinical manifestation was cough, followed by fever. Among the 29 patients, most had normal laboratory indicators. Procalcitonin and C-reactive protein levels increased in $9(31.0 \%)$ and $5(17.2 \%)$ of the patients, respectively, whereas the absolute number of lymphocytes was decreased in $6(20.7 \%)$ of the patients.

Based on the repeat CT scans, 10 (34.5\%) of the 29 patients developed new pulmonary lesions, whereas the

Table I Comparison of Clinical and Laboratory Information Between Patients with and without New Pulmonary Lesions

\begin{tabular}{|c|c|c|c|c|}
\hline Parameters & Total $(n=29)$ & $\begin{array}{l}\text { Patients with New } \\
\text { Lesions }(n=10)\end{array}$ & $\begin{array}{l}\text { Patients without New } \\
\text { Lesions }(n=19)\end{array}$ & $P$ value \\
\hline \multicolumn{5}{|l|}{ Sex } \\
\hline Male & $17(58.6)$ & $3(30.0)$ & 14 (73.7) & 0.046 \\
\hline Female & $12(41.4)$ & $7(70.0)$ & $5(26.3)$ & \\
\hline Age & $38.5 \pm 20.5$ & $51.1 \pm 13.3$ & $31.9 \pm 20.8$ & 0.024 \\
\hline \multicolumn{5}{|l|}{ Exposure History } \\
\hline Exposure to Infected Patients & $22(75.9)$ & $9(90.0)$ & $13(68.4)$ & 0.367 \\
\hline Work or Live in Wuhan & $5(17.2)$ & $I(10.0)$ & $4(2 I . I)$ & 0.633 \\
\hline Travel to Wuhan Recently & $2(6.9)$ & 0 & $2(10.5)$ & 0.532 \\
\hline \multicolumn{5}{|l|}{ Previous History } \\
\hline Hypertension & $2(6.9)$ & I $(10.0)$ & $\mathrm{I}(5.3)$ & 1.000 \\
\hline Hyperthyroidism & I (3.4) & I $(10.0)$ & 0 & 0.345 \\
\hline \multicolumn{5}{|l|}{ First Symptoms } \\
\hline Cough & $15(5 \mid .7)$ & $5(50.0)$ & $10(52.6)$ & 1.000 \\
\hline Fever & $9(31.0)$ & $5(50.0)$ & $4(2 I .1)$ & 0.205 \\
\hline Diarrhea & I (3.4) & 0 & I (5.3) & 1.000 \\
\hline Fatigue & I (3.4) & I $(10.0)$ & 0 & 0.345 \\
\hline No Obvious Symptoms & $6(20.7)$ & $2(20.0)$ & $4(2 I . I)$ & 1.000 \\
\hline \multirow{2}{*}{\multicolumn{5}{|c|}{$\begin{array}{l}\text { Laboratory Indicators (Increase/ } \\
\text { Normal/Decrease) }\end{array}$}} \\
\hline & & & & \\
\hline White Blood Cell Count & $2(6.9) / 26(89.7) / 1(3.4)$ & $0 / 10(100.0) / 0$ & $2(10.5) / 16(84.2) / 1(5.3)$ & 0.664 \\
\hline Neutrophil Count & $2(6.9) / 23(79.3) / 4(13.8)$ & $0 / 9(90.0) / 1(10.0)$ & $2(10.5) / 14(73.7) / 3(15.8)$ & 0.820 \\
\hline Neutrophil Percentage & $4(13.8) / 24(82.8) / I(3.4)$ & $2(20.0) / 8(80.0) / 0$ & $2(10.5) / 16(84.2) / /(5.3)$ & 0.364 \\
\hline Lymphocyte Count & $2(6.9) / 21(72.4) / 6(20.7)$ & $0 / 7(70.0) / 3(30.0)$ & $2(10.5) / 14(73.7) / 3(15.8)$ & 0.229 \\
\hline Lymphocyte Percentage & I (3.4)/20 (69.0)/8 (27.6) & $0 / 7(70.0) / 3(30.0)$ & I (5.3)/I $3(68.4) / 5(26.3)$ & 0.691 \\
\hline Procalcitonin & $9(31.0) / 20(69.0) /-$ & $4(40.0) / 6(60.0) /-$ & $5(26.3) / 14(73.7) /-$ & 0.625 \\
\hline C-Reactive Protein & $5(17.2) / 24(82.8) /-$ & $2(20.0) / 8(80.0) /-$ & $3(15.8) / 16(84.2) /-$ & 1.000 \\
\hline
\end{tabular}

Note: Data in parentheses are expressed as number (percentage) or mean \pm standard deviation. 
other 19 (65.5\%) cases still had no abnormality. Compared with patients without new lesions, those with new lesions were older and women were more common among them (Table 1).

\section{CT Manifestations of Newly Developed Lesions}

The average interval between the onset of new lesions and the initial chest CT scan was $5.8 \pm 3.0$ days, with a range of 2 to 12 days (2-4 days: 5 cases [50.0\%], 5-8 days: 4 cases [40.0\%], and $>8$ days: 1 case $[10.0 \%])$. And the average number of repeat CT scans was $4.0 \pm 1.4$ (range: $2-7$ ).

Among the 10 patients with newly developed lesions, 3 $(30.0 \%)$ of the patients had lesions involving the left lung; 1 (10.0\%), involving the right lung; and 6 (60.0\%), involving the bilateral lungs. The right upper lobe, the middle lobe, the lower lobe, the left upper lobe, and the lower lobe were involved in $2(20.0 \%), 3(30.0 \%), 6(60.0 \%), 4(40.0 \%)$, and $9(90.0 \%)$ of the patients, respectively. The numbers of patients with 1,2 , and $\geq 3$ involved lobes (mean: $2.5 \pm 1.6$, range: $1-5)$ were $4(40.0 \%), 3(30.0 \%)$, and $3(30.0 \%)$, respectively, and the numbers of patients with 1,2 , and $\geq 3$ involved segments (mean: $4.5 \pm 4.5$, range: $1-13$ ) were 5 $(50.0 \%), 1(10.0 \%)$, and $4(40.0 \%)$. All the new lesions presented as GGO on CT images without significant consolidation (Figures 1-3). The density was homogeneous in five lesions $(50.0 \%)$ and heterogeneous in five lesions (50.0\%), and the shape was spherical in two lesions $(20.0 \%)$, patchy in four lesions $(40.0 \%)$, and both spherical and patchy in four lesions $(40.0 \%)$.

\section{Changes in Patients' Clinical, Laboratory, and CT Manifestations During Treatment}

Cases with aggravated or new symptoms (6 cases) and decreased lymphocyte count (3 cases) in patients who developed new lesions were more common than those $(3$ and 0

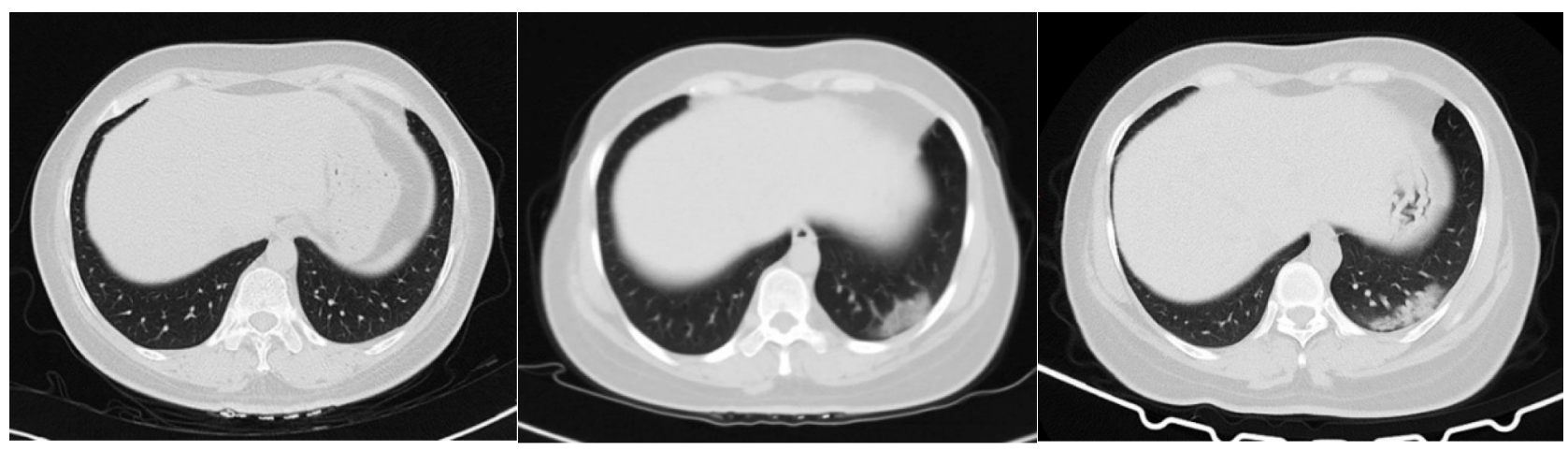

A

B

C
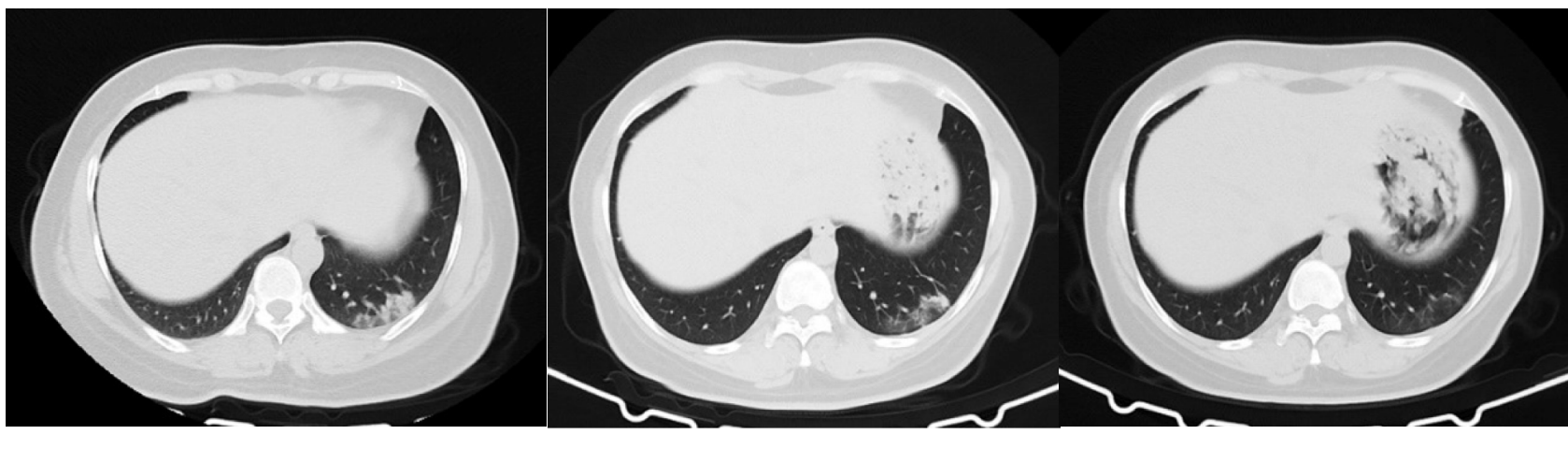

D

E

$\mathbf{F}$

Figure I A 45-year-old woman who exposed to COVID-19 patients has cough and diarrhea for 2 days. The initial chest CT is negative (A). After 4 days, the first repeat CT shows a new patch GGO located in the left lower lobe with homogeneous density (B). Subsequently, this new lesion increases in extent and density (C), and then absorbs gradually ( $\mathbf{D}$ and $\mathbf{E})$. On the latest CT scan (31 days later), it significantly absorbs and leaves a small amount of faint GGO (F). 


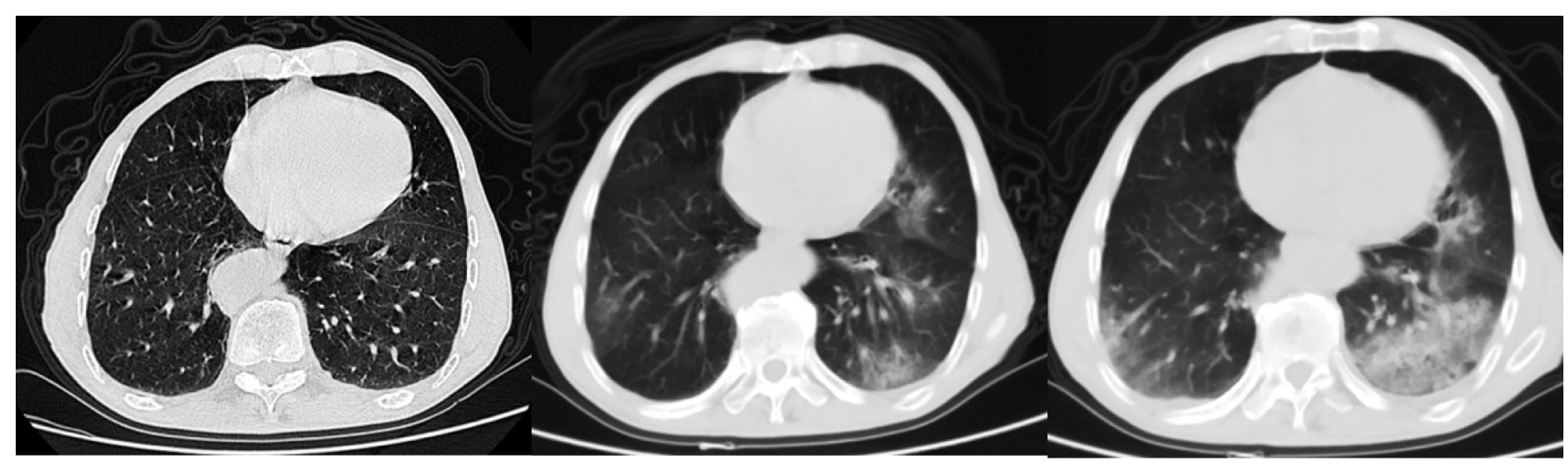

A

B

C

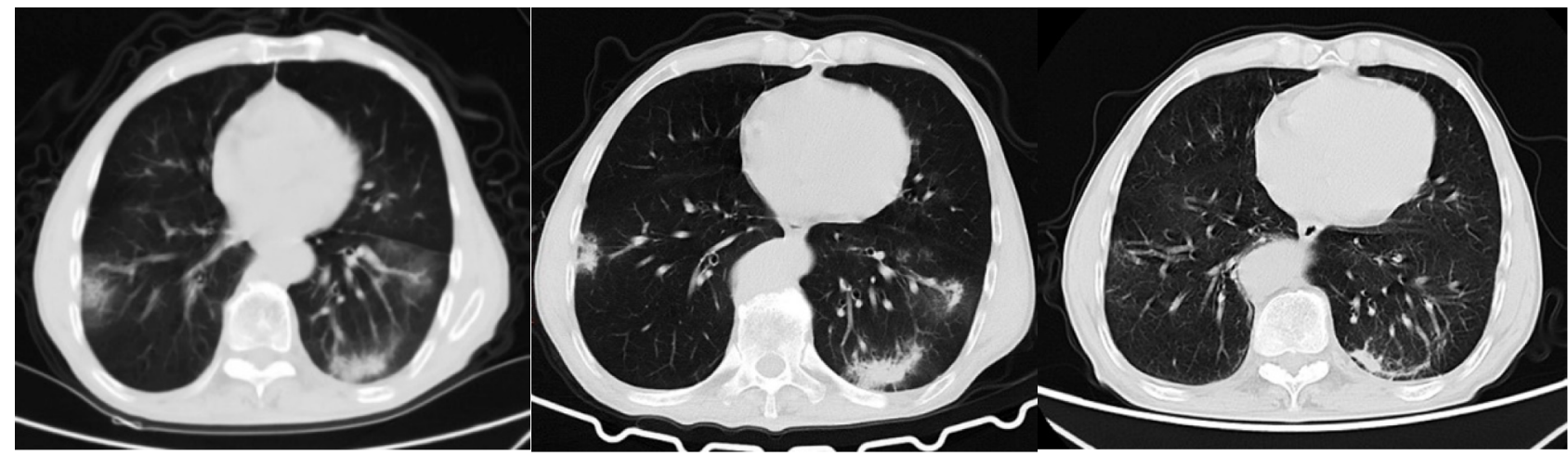

D

E

F

Figure 2 A 73-year-old man who exposed to COVID-19 patients has fever and fatigue for 2 days. The initial chest CT is negative (A). After 2 days, the first repeat CT shows multiple patchy GGOs with heterogeneous density developed in the bilateral lungs (B). Subsequently, some of new lesions increase in extent and density (C), and then absorbs gradually with decrease of extent and increase of density (D and $\mathbf{E}$ ). On the latest CT scan (38 days later), they significantly absorb and leave few fibrous strips (F).

cases) in patients who did not develop new lesions $(60.0 \%$ vs $15.8 \%, \mathrm{P}=0.032,30.0 \%$ vs $0.0 \%, \mathrm{P}=0.033$, respectively). The number of involved lung segments in 4 patients without symptoms was 1 to 2 , with an average of $1.5 \pm 0.6$ segments, whereas the 6 patients with worsening or new symptoms had 1 to 13 involved segments, with an average of $6.5 \pm 5.0$ segments $(1.5 \pm 0.6$ vs $6.5 \pm 5.0, \mathrm{P}=0.057)$. During treatment, new lesions in $4(40.0 \%)$ of the patients were directly absorbed (Figure 3), whereas those in $6(60.0 \%)$ of the patients progressed (increasing in extent or density) and were then absorbed gradually (Figures 1 and 2). On the latest CT scan, residual fibrous strips with or without GGO (Figure 2F), solely faint GGO (Figures $1 \mathrm{~F}$ and $3 \mathrm{~F}$ ), and no residue were found in 5 $(50.0 \%), 4(40.0 \%)$, and $1(10.0 \%)$ of the patients, respectively.

\section{Discussion}

The epidemic of COVID-19 has attracted much attention because of its harm to health and life. The risk of expansion is due to the long latent period, the clinical symptoms are not easy to be identified and the hiddenness of infection is higher, which increases the probability of disease transmission. Moreover, in view of the disadvantages of RT-PCR and CT scanning in identifying and following up the disease, it is necessary to combine laboratory test results, clinical symptom development and CT changes to evaluate the disease comprehensively.

In the present study, some patients developed new pulmonary lesions during treatment, although most patients' repeat CT scans were still negative. Cases with aggravated or new symptoms and abnormal laboratory indicators in patients who developed new lesions were more common than those in patients who did not develop new lesions. It indicates that the occurrence of new lesions in some COVID-19 patients may be accompanied by the deterioration of clinical manifestations or laboratory indicators. In addition, changes in clinical symptoms may be 


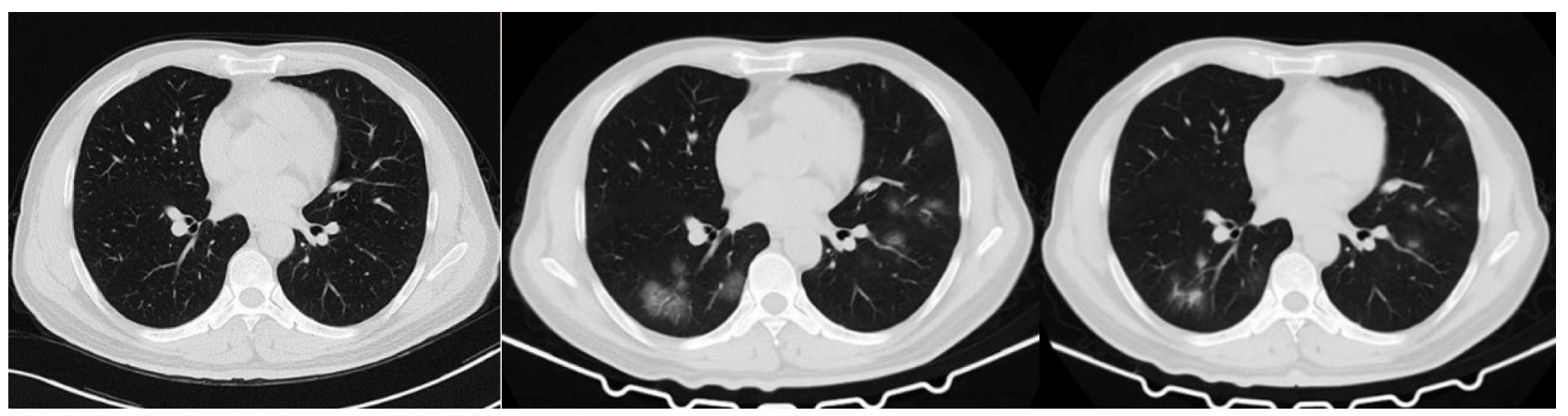

A

B

C

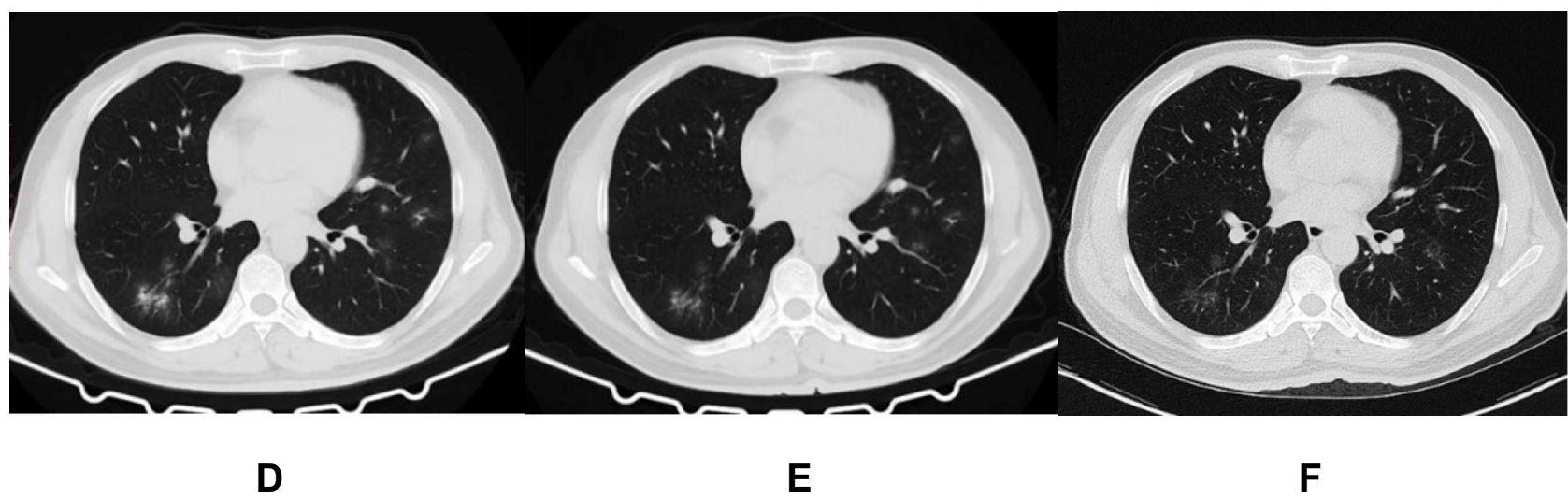

Figure 3 A 44-year-old man who exposed to COVID- 19 patient has fever, cough, and diarrhea for 4 days. The initial chest CT is negative (A). After 7 days, the first repeat CT shows multiple spherical and patchy GGOs with heterogeneous density developed in the bilateral lungs (B). Subsequently, the lesions directly absorb with decrease of extent and temporary increase of density (C-E). On the latest CT scan (29 days later), they significantly absorb and leave a small amount of faint GGO (F).

related to the extent of pulmonary involvement since patients with aggravated or new symptoms typically had relatively more involved lung segments than asymptomatic cases. Therefore, a repeat chest CT scan is necessary for patients who are negative by initial CT screening, especially for those with worsening or new symptoms during treatment.

Four stages of lung involvement were defined in the analysis of the CT progression of COVID-19 patients: early stage (within 4 days after the initial symptom), progressive stage (5-8 days), peak stage (9-13 days), and absorption stage ( $\geq 14$ days). ${ }^{14}$ It is worth mentioning that patients in this study typically had no pulmonary lesions in the early stage or throughout the entire course of the disease. This might be because their lungs were not affected or because early lesions were well controlled by treatment before displaying on $\mathrm{CT}$ images. This demonstrates that chest $\mathrm{CT}$ might provide false-negative results in special groups. ${ }^{15}$ In addition, there is a certain degree of false-negative results with the use of the RT-PCR test. $^{12,16,17}$ Therefore, it is necessary to appropriately combine the results of RT-PCR and chest $\mathrm{CT}$ for patients with suspected COVID-19 infection.

In this study, the newly developed lesions were typically located in the lower lobes with spherical or patchy GGO and without significant consolidation, which was consistent with the CT manifestations of early-stage lesions. ${ }^{18-20}$ During follow-up, new lesions in $60.0 \%$ of the patients progressed with an increase in extent and/or density, but all lesions were eventually significantly absorbed. This indicates that close follow-up CT scans are necessary for these patients to determine the occurrence of and change in new lesions. This is helpful for quickly adjusting the treatment strategy of individual patients and for better control of the development of new lesions.

This study had two limitations. First, the samples were relatively small because most patients already have pulmonary lesions at the time of consultation, which may make some statistical differences not reasonable. Second, although the patients had negative chest $\mathrm{CT}$, they were immediately treated after diagnosis. Thus, the present 
results do not represent the natural development process, because some lesions may have been controlled before they are displayed on $\mathrm{CT}$.

\section{Conclusion}

In conclusion, some COVID-19 patients with initial negative chest CT will develop new pulmonary lesions during treatment. The newly developed lesions may be localized or extensive, and most will temporarily progress but significantly be absorbed subsequently. Repeat CT is necessary for monitoring the disease in such patients, especially when they have worsening symptoms or laboratory indicators.

\section{Funding}

This study was supported by New Coronavirus Pneumonia Emergency Clinical Research project in Chongqing Medical University and New Coronavirus Pneumonia Emergency project in Science and Technology Bureau of Yongchuan District (No. ycstc,2020nb0204).

\section{Disclosure}

The authors report no conflicts of interest in this work.

\section{References}

1. Li Q, Guan X, Wu P, et al. Early transmission dynamics in Wuhan, China, of novel coronavirus-infected pneumonia. $N$ Engl $J$ Med. 2020;382:1199-1207. doi:10.1056/NEJMoa2001316

2. Zhu N, Zhang D, Wang D, et al. A novel coronavirus from patients with pneumonia in China, 2019. N Engl J Med. 2020;382:727-733. doi:10.1056/NEJMoa2001017

3. Mahase E. China coronavirus: WHO declares international emergency as death toll exceeds 200. BMJ. 2020;368:m408. doi:10.1136/bmj. $\mathrm{m} 408$

4. Xu X, Chen P, Wang J, et al. Evolution of the novel coronavirus from the ongoing Wuhan outbreak and modeling of its spike protein for risk of human transmission. Sci China Life Sci. 2020;63:457-460. doi:10.1007/s11427-020-1637-5

5. Wan Y, Shang J, Graham R, Baric RS, Li F. Receptor recognition by the novel coronavirus from Wuhan: an analysis based on decade-long structural studies of SARS coronavirus. $J$ Virol. 2020;94. doi:10.1128/ JVI.00127-20
6. Xu X, Wu X, Jiang X, et al. Clinical findings in a group of patients infected with the 2019 novel coronavirus (SARS-Cov-2) outside of Wuhan, China: retrospective case series. BMJ. 2020:m606. doi:10.1136/bmj.m606

7. Chen N, Zhou M, Dong X, et al. Epidemiological and clinical characteristics of 99 cases of 2019 novel coronavirus pneumonia in Wuhan, China: a descriptive study. Lancet. 2020;395:507-513. doi:10.1016/S0140-6736(20)30211-7

8. Wang F, Zhang C. What to do next to control the 2019-nCoV epidemic? Lancet. 2020;395:391-393. doi:10.1016/S0140-6736(20) 30300-7

9. World Health Organization. Situation report - 81. 10 April 2020. Available from: https://www.who.int/docs/default-source/coronavir use/situation-reports/20200410-sitrep-81-covid-19.pdf?sfvrsn= ca96eb84_2. Accessed July 23, 2020.

10. Chan JF, Yuan S, Kok K, et al. A familial cluster of pneumonia associated with the 2019 novel coronavirus indicating person-toperson transmission: a study of a family cluster. Lancet. 2020;395:514-523. doi:10.1016/S0140-6736(20)30154-9

11. Fang X, Zhao M, Li S, Yang L, Wu B. Changes of CT findings in a 2019 novel coronavirus $(2019-\mathrm{nCoV})$ pneumonia patient. $Q J M$. 2020;113:271-272. doi:10.1093/qjmed/hcaa038

12. Ai T, Yang Z, Hou H, et al. Correlation of chest CT and RT-PCR testing in coronavirus disease 2019 (COVID-19) in China: a report of 1014 cases. Radiology. 2020:200642.

13. Xie X, Zhong Z, Zhao W, Zheng C, Wang F, Liu J. Chest CT for typical 2019-nCoV Pneumonia: relationship to negative RT-PCR testing. Radiology. 2020:200343.

14. Pan F, Ye T, Sun P, et al. Time course of lung changes on chest CT during recovery from 2019 novel coronavirus (COVID-19) pneumonia. Radiology. 2020:200370.

15. Bernheim A, Mei X, Huang M, et al. Chest CT findings in coronavirus disease-19 (COVID-19): relationship to duration of infection. Radiology. 2020;295:200463. doi:10.1148/radiol.2020200463

16. Yang Y, Yang M, Shen C, et al. Evaluating the accuracy of different respiratory specimens in the laboratory diagnosis and monitoring the viral shedding of 2019-nCoV infections. medRxiv. 2020. doi:10.1101/ 2020.02.11.20021493

17. Fang Y, Zhang H, Xie J, et al. Sensitivity of chest CT for COVID-19: comparison to RT-PCR. Radiology. 2020:200432.

18. Song F, Shi N, Shan F, et al. Emerging 2019 novel coronavirus (2019-nCoV) pneumonia. Radiology. 2020;295:210-217. doi:10.11 48/radiol.2020200274

19. Pan Y, Guan H, Zhou S, et al. Initial CT findings and temporal changes in patients with the novel coronavirus pneumonia (2019-nCoV): a study of 63 patients in Wuhan, China. Eur Radiol. 2020;30(6):3306-3309. doi:10.1007/s00330-020-06731-x

20. Chung M, Bernheim A, Mei X, et al. CT imaging features of 2019 novel coronavirus (2019-nCoV). Radiology. 2020;295:202-207. doi:10.1148/radiol.2020200230
Infection and Drug Resistance

\section{Publish your work in this journal}

Infection and Drug Resistance is an international, peer-reviewed openaccess journal that focuses on the optimal treatment of infection (bacterial, fungal and viral) and the development and institution of preventive strategies to minimize the development and spread of resistance. The journal is specifically concerned with the epidemiology of antibiotic resistance and the mechanisms of resistance development and diffusion in both hospitals and the community. The manuscript management system is completely online and includes a very quick and fair peerreview system, which is all easy to use. Visit http://www.dovepress.com/ testimonials.php to read real quotes from published authors. 